\title{
ESTRATEGIAS DE FINANCIAMIENTO, UN RETO PARA LAS PYMES COMERCIALES DE GUANACASTE
}

\author{
FINANCING STRATEGIES, A CHALLENGE FOR COMMERCIAL SMES \\ IN GUANACASTE
}

Héctor Canossa-Montes de Oca ${ }^{1}$

Roberto Rodríguez-Alcocer ${ }^{2}$

Recibido: 13.08 .19

Aprobado: 26.11.19

DOI: $10.15517 /$ isucr.v20i42.41845

\section{Resumen}

Las empresas requieren una óptima administración financiera para permanecer y crecer en el mercado, según estudios de Costa Rica ocho de cada diez empresas no sobreviven más de diez años, aunado a que Guanacaste contabiliza únicamente el cinco por ciento de total de pymes del país, por lo que se requieren nuevos emprendimientos y la consolidación de las actuales para hacer crecer este segmento empresarial y por ende contribuir con el desarrollo económico de la provincia. Este artículo tiene como objetivo analizar retos para el financiamiento en las empresas estudiando la perspectiva de las pymes comerciales de Guanacaste. Los resultados obtenidos incluyen que el sector analizado ha desarrollado sus negocios a través de un financiamiento personal con ahorros, o incluso usando recursos provenientes de otro empleo y proveedores, y en su mayoría los recursos sumados de inversión inicial no superan los cinco millones de colones; pero el deseo de expansión requiere que estas empresas realicen un análisis y evaluación del endeudamiento, como fuente de inversión para posicionarse en el mercado, y para la mayoría los temas financieros son desconocidos. Estas empresas deben reconocer la relevancia de la gestión financiera y buscar estrategias para crecer mediante un constante aprendizaje con capacitaciones sobre financiamiento y administración en general. Esta investigación promueve una orientación hacía la gestión financiera de una manera responsable, buscando la rentabilidad y el crecimiento de las pymes comerciales de Guanacaste y Costa Rica.

\section{Palabras clave}

\footnotetext{
${ }^{1}$ Costarricense. Profesor Universidad Técnica Nacional, Sede Guanacaste. Email: hcanossa@utn.ac.cr

${ }^{2}$ Costarricense. Profesor Universidad de Costa Rica, Sede Guanacaste y Universidad Técnica Nacional, Sede Guanacaste. Email: ROBERTO.RODRIGUEZALCOCER@ucr.ac.cr
} 
Financiamiento; estrategia; administración; pymes; Guanacaste.

\section{Abstract}

Companies require optimal financial management to remain and grow in the market, eight out of ten companies do not survive more than ten years and only 5 percent of SMEs in Costa Rica are found in Guanacaste, so its required more consolidated companies in the area that contribute to economic development. This article aims to analyze challenges for financing in companies by studying the perspective of commercial SMEs in Guanacaste. The results obtained include that the analyzed sector has developed its business through personal financing with savings, or even using resources from other employment and, in addition, making initial investments of less than five million and with the support of suppliers have managed to position their SMEs, although very few expanding in the market, on this expansion show desires to do it even through indebtedness, but affirm ignorance in financial matters. These companies must recognize the relevance of financial management and seek strategies to grow through constant learning with training on financing and administration in general. This research promotes an orientation towards management in a responsible manner, seeking the profitability and growth of commercial SMEs in Guanacaste and Costa Rica.

\section{Keywords}

Financing; strategy; administration; SMEs; Guanacaste.

\section{Introducción}

El financiamiento es un tema muy importante para todos los seres humanos, porque se requiere ser eficientes en la administración de los recursos, fuente primordial para la obtención de ingresos, a través de productos y servicios que satisfagan las necesidades del mercado; esto también es aplicable a las diversas empresas que se encuentran en el entorno, ya que estas necesitan una forma de financiarse y tomar decisiones sobre el uso del dinero.

Un dato inicial relevante es que ocho de cada diez empresas no sobreviven más de diez años, esto lo indica Marcelo Lebendiker, presidente de Parque Tec (citado por Pymes, El Financiero, 2015) y algunas de las razones es la falta conocimiento y no tener un acceso óptimo a financiamiento, es así como este aspecto recobra la relevancia de estudiar y aprender sobre temas financieros y son la base de la toma de decisiones.

En Guanacaste es necesario realizar aportes que promuevan a las empresas comerciales la posibilidad de sobrevivir y crecer en el mercado; entre más empresas y más consolidadas estén, será mejor para la provincia, ya que estas generan más empleos, ingresos y posibilidades de bienestar para la población. Esta investigación trata de un análisis y propuesta de estrategias de financiamiento para que las pymes comerciales de Guanacaste puedan adquirir recursos productivos, y estos contribuyan para mantenerse y desarrollarse en el mercado.

Para ello, se plantea la siguiente pregunta: ¿por qué y para qué se debe desarrollar este tema de investigación? La respuesta sería, que las finanzas son una función clave de toda empresa; implican, en pocas palabras, la obtención de dinero por medio de los ingresos y financiamiento, la colocación de este en inversiones, costos y/o gastos, así como los resultados que se obtienen a través de este proceso, por lo tanto, este tema es importante para estudiar el financiamiento como una fuente de obtención de recursos productivos, y si previo se utilizaron las herramientas 
adecuadas de análisis, la ruta de consolidación y crecimiento de las empresas aumenten las posibilidades de sobrevivencia y creación de valor.

Por consiguiente, el tema sería de utilidad para las pequeñas y medianas empresas comerciales que están en el mercado de Guanacaste y que desean crecer a través de la adquisición eficiente de los activos o recursos productivos por medio del financiamiento, eso sí, este financiamiento sería únicamente para la obtención de activos de inventarios, bienes muebles e inmuebles necesarios para la operación de la empresa, y previo a la decisión debería existir un análisis de escenarios considerando las diversas opciones existentes en el mercado para lograr materialización la gestión de estos recursos al menor costo financiero.

Los beneficios de esta investigación incluyen la identificación de cómo se han financiado a la fecha las pymes guanacastecas, a través del conociendo de la situación actual, esto permite la generación de propuestas de mejoraras en el tema de estrategias de la administración financiera, sobre la búsqueda de hacerlas más competitivas.

El aporte es un análisis de las condiciones actuales de las empresas comerciales de Guanacaste, una provincia del país que históricamente ha estado aislado de la Gran Área Metropolitana; por lo tanto, se busca aportar al crecimiento de las empresas de esta región del país y el bienestar de sus habitantes.

Una vez definida la utilidad, beneficios y aporte de la investigación, se plantea el siguiente problema: ¿cuáles son las posibles estrategias de financiamiento para la obtención de recursos productivos que las pymes comerciales de Guanacaste pueden implementar durante el segundo semestre de 2019?

Para dar respuesta al problema se propone como objetivo general, analizar las posibles estrategias de financiamiento para la obtención de recursos productivos que las pymes comerciales de Guanacaste requieren.

Además, específicamente, se pretende identificar las necesidades de financiamiento que las pymes en estudio tienen en el corto plazo; describir las fuentes para el financiamiento de los recursos productivos que las pymes tienen a disposición para la creación de la estrategia y sugerir las estrategias de financiamiento que las pymes comerciales de Guanacaste pueden utilizar en el corto plazo para el crecimiento de sus operaciones.

Para lograr el cumplimiento de los objetivos definidos de la investigación, se fundamenta en un trabajo de campo donde se estudia una muestra de pymes comerciales guanacastecas; posteriormente, se realiza un análisis de la investigación o discusión de resultados y se finaliza con las conclusiones y recomendaciones de la investigación.

Revisión Bibliográfica

El análisis de las estrategias de financiamiento para las empresas es muy importante, su adecuada ejecución influye en su permanencia en el mercado y las posibilidades de crecimiento, donde el entorno es cada día más competitivo, globalizado y con una gran influencia de las tecnologías de información y comunicación. Como explica Chiavenato (2017), la estrategia organizacional surge como consecuencia de la intensa competencia entre organizaciones y el 
proceso de planeación estratégica es la herramienta que busca las ventajas competitivas de la empresa, para alcanzar los objetivos (pág. 1).

Precisamente estas estrategias orientadas al financiamiento son las que facilitan la obtención de recursos productivos, o en términos contables llamados activos, que incluyen los recursos físicos que una empresa puede poner a trabajar para alcanzar objetivos del negocio (Harvard Business Review, 2017, pág. 10).

Estos recursos son los que les permiten a las empresas comerciales su operación y la posibilidad de satisfacer las necesidades y expectativas de los clientes y diversos interesados; por lo tanto, estas estrategias deberán ser dirigidas a través de una adecuada administración. Por estos aspectos, también se hace relevante reconocer la definición y funciones que tiene la administración financiera; como explica Salas (2016):

Se define como la encargada de planear, evaluar, captar y controlar los fondos generados y requeridos de las diversas fuentes de efectivo y financiamiento, así como planificar, dirigir y controlar los recursos aplicados e invertidos en forma eficiente y oportuna, con el propósito de cumplir con todas las obligaciones y compromisos de su operación y poder satisfacer adecuadamente los objetivos, planes y actividades del negocio, orientados a maximizar el valor de la empresa en el tiempo. (pág. 21)

Para cumplir estos objetivos, los recursos se pueden financiar por dos fuentes, sea recursos propios; es decir, el capital o patrimonio de los dueños del negocio, o bien con endeudamiento o en términos contables llamados pasivos; para este análisis se utiliza el estado financiero conocido como balance general o estado de situación financiera que incluye las secciones por una parte de activos o recursos y en la otra parte las fuentes de financiamiento que son pasivos y/o patrimonio.

Ross, Westerfield, Jaffe y Jordan (2018) explican sobre el estado de situación financiera:

Es una fotografía instantánea (tomada por un contador) del valor contable de una empresa en una fecha determinada, como si la empresa se quedara momentáneamente inmóvil. El estado de situación financiera tiene dos lados: en el lado izquierdo están los activos, mientras que en el derecho se encuentran los pasivos y el capital contable. El estado de situación financiera muestra lo que la empresa tiene y la manera en que se financia. (pág. 20).

Estos recursos productivos, y la forma en que se financian, son esenciales para que las operaciones de la empresa generen resultados adecuados y crezcan, ya que, por ejemplo, los altos niveles de endeudamiento podrían generar mayores gastos financieros que afectan los resultados, en términos de ganancias y crecimiento de la entidad; esta investigación plantea el análisis enfocándose en las pymes comerciales de Guanacaste, sobre el concepto de las Pymes son las unidades productivas que dispones de recursos humanos y los gestiona, bajo las figuras de persona física o jurídica, en actividades industriales, comerciales, de servicios o agropecuarias de agricultura orgánica (Pymes Costa Rica, 2019, párr. 1).

Además, considerando el componente comercial que se pretende estudiar, Carrasco (2017) define las empresas comerciales como las que se dedican a la venta directa de productos a los 
consumidores (pág. 4), esto es importante por la delimitación de la investigación de pymes comerciales donde, además, geográficamente se ubica en Guanacaste.

En Guanacaste, considerando los datos del Directorio de Empresas y Establecimientos (DEE) del INEC (2019), existen unas 1.691 empresas tomando el intervalo de trabajadores que no superen los cien ya que a este punto se considerarían grandes empresas; cabe agregar que 34,7 por ciento de las empresas se dedican a actividades comerciales; es decir, 587 son pymes comerciales. Como dato importante a nivel de Costa Rica las empresas que se incluyen en el Directorio de Empresas y Establecimientos muestran que en Guanacaste solo se encuentra el 5 por ciento de las 35.429 empresas a nivel del país, tal como se muestra en la figura 1.

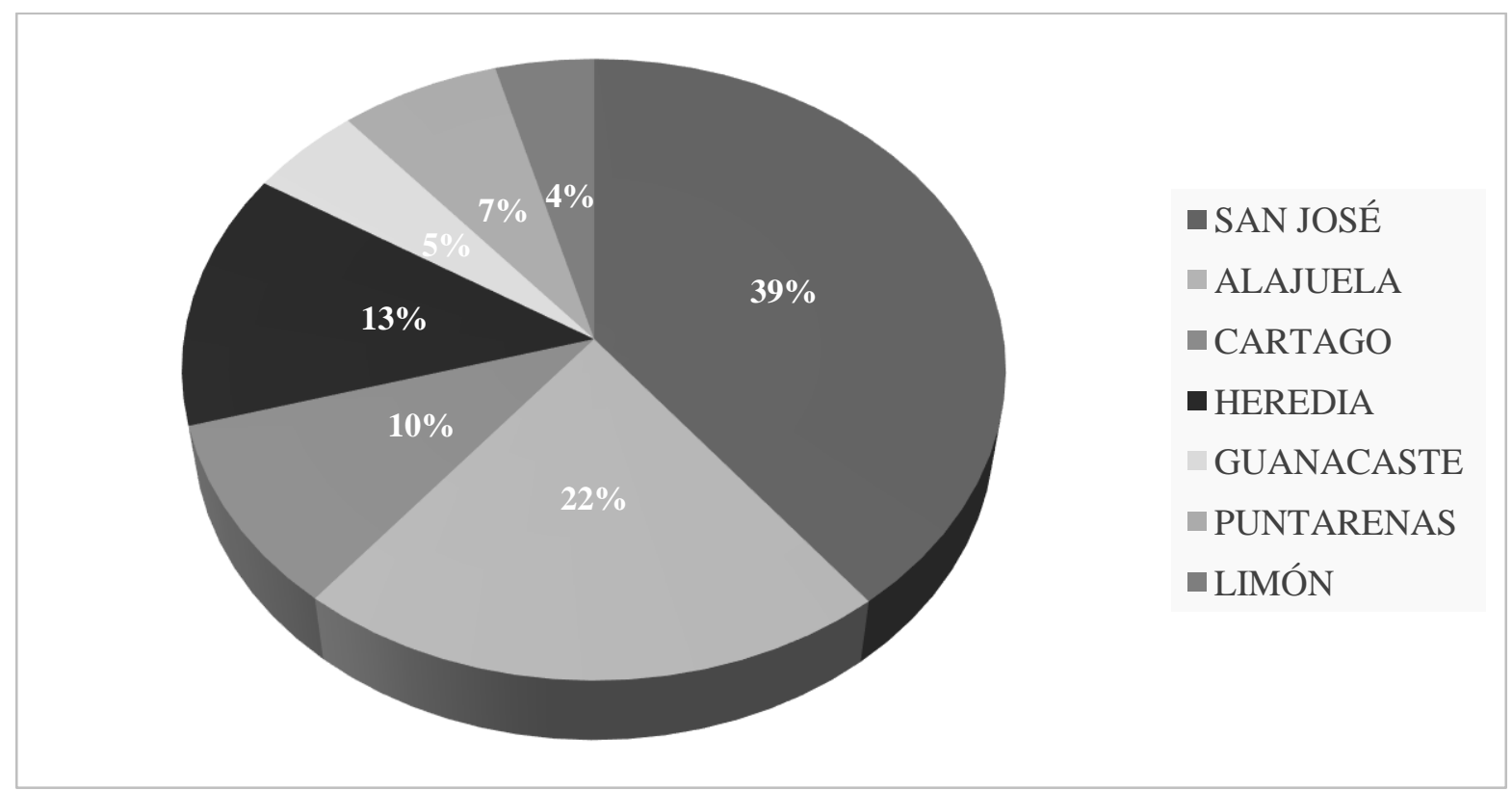

Figura 1. Porcentajes según la provincia de las 35.429 pequeñas y medianas empresas registradas en el Directorio de Empresas y Establecimientos del INEC en Costa Rica. (Fuente: elaboración propia basada en el Directorio de Empresas y Establecimientos, INEC, 2019).

Una vez identificado el porcentaje de pymes guanacastecas, se aborda el tópico de reconocer las necesidades de financiamiento que tienen y la obtención de recursos o activos que pueden incluir desde los inventarios para generar ingresos a corto plazo, incluso aumentar la capacidad de la empresa a través de equipos, vehículos, tecnología o adquisición de puntos de venta. Sobre este tema, Salas (2016) afirma que

los incrementos de activos constituyen usualmente el principal empleo de recursos en las empresas, ya que sus actividades comerciales, productivas y administrativas imponen aumentar o renovar sus inversiones en activos circulantes y a largo plazo. El crecimiento y la expansión de las operaciones demandan incrementar el capital de trabajo y aumentar la capacidad instalada, así como nuevas inversiones en tecnología y sistemas. (pág. 204)

Por otra parte, una fuente de financiamiento de estos recursos productivos o activos, son las propias ganancias que van obteniendo las empresas por sus actividades; es decir, utilidades 
retenidas, también, se pueden considerar los aportes de capital que hacen los propietarios, y la tercera opción la componen el endeudamiento con créditos pymes, créditos fiduciarios, hipotecarios y prendarios y otros instrumentos financieros como leasing y renting e inventarios en consignación.

Gutiérrez (2017) expone que muchas pymes tienen la intención de invertir en maquinaria, equipo, insumo o cualquier otro aspecto que incremente su productividad, pero no cuentan con el capital; para hacerlo ahí entran en escena los bancos públicos y privados que ofrecen programas especiales, con tasas de interés diferenciadas dirigidas al capital de trabajo, inversiones amigables con el ambiente y la compra de vehículos nuevos, además las tasas varían entre el 7,25 por ciento y el 13,25 por ciento dependiendo del programa, el plazo y el tipo de garantía.

Se recalca que la decisión de financiarse se centra en dos principales formas de obtener los recursos financieros: capital aportado por los propietarios y utilidades retenidas o por deudas o pasivos; en la figura 1 se muestran las formas en que se puede subdividir estas fuentes de financiamiento, que incluye las ganancias del mismo negocio, los ahorros personales o ingresos por otro empleo, así como los aportes de familia y amigos, al ser empresas comerciales también los proveedores que dan mercadería en consignación, como otra opción los créditos o subsidios apoyados por programas del gobierno y otras opciones como inversionistas que aportan capital, instituciones financiera como bancos que otorgan créditos y el uso de las tarjetas de crédito.

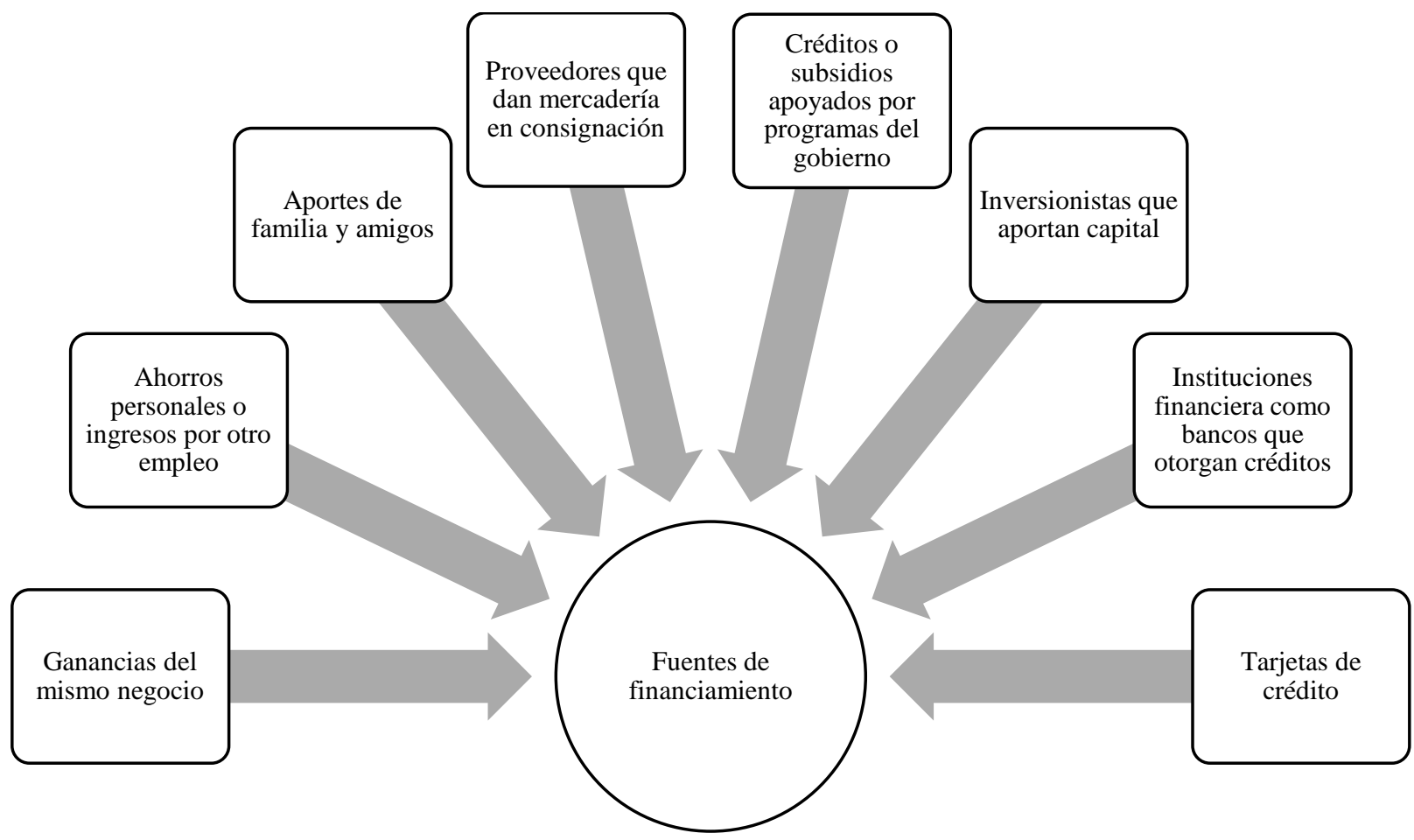

Figura 2. Fuentes de financiamiento que pueden usar las pymes comerciales de Guanacaste para la obtención de recursos productivos. (Fuente: elaboración propia).

Las pymes deben considerar al momento de la obtención de recursos por medio de préstamos o pasivos, las variables que estos incluyen tales como; tipo de crédito, la garantía (por ejemplo: fiador, hipoteca, prenda), la cantidad de dinero, el plazo que normalmente a mayor plazo la cuota 
disminuye, la tasa de interés que dependerá en mucho de la garantía y riesgo, la amortización y gastos de formalización; cabe agregar que según la herramienta de financiamiento que se utilice puede implicar mayores o menores costos para la empresa y, por tanto, tener repercusiones en sus expectativas de ganancias.

Para las pymes la obtención de financiamiento a corto plazo se debe considerar; es pensar en las inversiones en activos con endeudamiento que no superen un año de plazo, generalmente para adquirir también activos corrientes de corto plazo o de la operación normal de la empresa. Ramírez (2018) aporta que para la buena toma de decisiones a corto plazo se debe: reconocer y definir el problema o necesidad de financiamiento; identificar posibilidades como posibles soluciones; determinar los costos y beneficios de cada una de las posibilidades; clasificar lo relevante o irrelevante; considerar factores cualitativos y, finalmente, seleccionar la opción que ofrezca la mayor ganancia (pág. 188).

Para concretar, estas pymes comerciales pueden buscar el crecimiento de las operaciones a través de estrategias que les permitan ser más competitivas al aprovechar las oportunidades que se presentan en el mercado; para esto las pymes deberán estar preparadas para encontrar formas efectivas de financiar este crecimiento y la adquisición de recursos productivos, a través de una adecuada administración empresarial y financiera.

Algunas estrategias empresariales incluyen: la integración de nuevas actividades productivas relacionadas con la producción o distribución (por ejemplo: la adquisición de vehículos para hacer entregas a domicilio); así como la diversificación, implica aumento de las líneas de productos, inventarios o desarrollos de negocios complementarios que apoyen el incremento de las ventas o bien estrategias de desarrollo de mercado que implica la expansión en nuevas áreas geográficas, como explican Thompson et al (2018) "las ofensivas estratégicas son necesarias cuando una organización encuentra oportunidades de ganar una participación de mercado rentable” (pág. 208).

\section{Metodología}

El tipo de investigación es descriptiva, Hernández, Fernández y Baptista (2014) explican que estos estudios buscan especificar propiedades y características importantes del fenómeno que se analiza, describiendo tendencias de una población (pág. 92). En el trabajo se busca analizar las características, tendencias y estrategias de las pymes comerciales de Guanacaste en relación con el financiamiento de recursos productivos que tienen actualmente y las estrategias aplicables. En el estudio se desarrolla una base teórica y, posteriormente, se pretende describir los comportamientos de las pymes comerciales guanacastecas.

La población investigada es finita e incluye el conjunto de todas las pymes comerciales de Guanacaste registradas en el Directorio de Empresas y Establecimientos del INEC que incluyen 587 empresas ubicadas en los once cantones de la provincia (INEC, 2019).

La población es el conjunto de todos los casos que concuerdan con características. Mientras que la muestra es el subgrupo de esa población del cual se recolectan los datos y que debe ser representativo de ésta (Hernández et al., 2014, pág. 173-174). En este punto es importante acotar la cantidad de participantes de la investigación para el cumplimiento óptimo de los objetivos de la investigación. 
La muestra corresponde a 83 pymes comerciales guanacastecas, utilizando un muestreo probabilístico simple con un nivel de confianza del 90 por ciento y un margen de error del 5 por ciento. Tomando en cuenta el tratamiento de los datos de los participantes se les indicó que la información aportada sería confidencial y utilizada para la presente investigación.

La técnica elegida para el estudio es una encuesta y como instrumento para la recolección de datos se utilizó un cuestionario de 14 preguntas para aportar a los objetivos específicos de la investigación; además, en estas se usan interrogantes de selección múltiple, así como la conocida escala de Likert para conocer los niveles de acuerdo con la afirmación de los encuestados; a esto se suman las preguntas de perfil del encuestado, buscando dar validez y lograr una discusión más adecuada en la investigación.

Análisis de la Investigación

La etapa de análisis de la investigación permite el desarrollo de la discusión de resultados al convertir los datos obtenidos de la encuesta en información y conocimientos sobre el tema en estudio.

El cuestionario fue contestado por los encargados o propietarios de las pymes comerciales de Guanacaste, y se obtiene como perfil del encuestado que el 62 por ciento son hombres y 38 por ciento son mujeres, además el 52 por ciento tienen de 41 años en adelante, 42 por ciento entre 25 y 41 años y solamente el 6 por ciento tiene entre 18 y 25 años. Otros datos revelan que los comercios encuestados se dedican en general a la venta de prendas de vestir, productos de salud y alimentos, el 60 por ciento, y a nivel de estudios solo completó la secundaria la cuarta parte y un 28 por ciento poseen estudios universitarios completos.

Sobre los hallazgos en primer lugar, como se muestra en el cuadro 1, al preguntar de dónde el obtuvo el financiamiento para iniciar las operaciones, la mayoría, es decir un 48 por ciento, indicó que el dinero se originó de ahorros personales y en segundo lugar un 32 por ciento de los encuestados afirmó que el negocio fue financiado con ingresos provenientes de otro empleo.

Las fuentes de financiamiento menos usadas para iniciar correspondieron a los préstamos con institución financiera con solo 6 por ciento y aportes de socios o inversionistas con 4 por ciento de las respuestas de los encuestados. Los encuestados hicieron comentarios sobre que para iniciar los negocios es difícil que una institución financiera los califique, ya que requieren demostrar que el negocio funciona y, en muchas ocasiones, exigen tener años de experiencia.

\section{Cuadro 1}

Forma de financiamiento que las pymes comerciales guanacastecas utilizaron para iniciar sus operaciones

\begin{tabular}{lll}
\hline & Cantidad & Porcentaje \\
\hline Ahorros personales & 40 & $48 \%$ \\
\hline Financiado con ingresos por otro empleo & 27 & $32 \%$ \\
\hline Préstamo con una institución financiera & 8 & $10 \%$ \\
\hline Aportes de socios o inversionistas & 5 & $6 \%$ \\
\hline Aportes de familiares o amigos & 3 & $4 \%$ \\
\hline Total & 83 & $100 \%$ \\
\hline
\end{tabular}


Fuente: Elaboración propia.

En relación con la inversión inicial requerida para el inicio de las operaciones de la empresa, al consultar a los encuestados sobre la cantidad de dinero requerida, la mayoría, es decir un 68 por ciento, necesitó entre más de un millón y cinco millones de colones, solamente un 8 por ciento de las pymes encuestadas requirió más de cinco millones de colones. Este es un dato interesante ya que 92 por ciento de las empresas en estudio lograron iniciar operaciones con menos de cinco millones, generando una idea de lo requerido para emprender.

Ante la consulta sobre las áreas de crecimiento de las empresas posterior al primer año de operación, 86 por ciento de los encuestados, indican que crecieron con más mercadería, además 62 por ciento de los encuestados contrataron más personal, 22 por ciento adquirieron activos fijos, $\mathrm{y}$ se destaca que solo 8 por ciento de las pymes consultadas abrieron un nuevo punto de venta.

Los resultados evidencian que las empresas están creciendo en sus operaciones normales con la adquisición de inventarios, pero no, con la apertura de nuevos negocios en el mercado, la justificación se sustenta en el comentaron del temor de abrir un nuevo punto de venta, a raíz de la incertidumbre de la aceptación o no del mercado, ya que afirman que no ha sido fácil el crecimiento actual, y consideran el riesgo de descuidar el negocio presente, el cual, ha logrado un posicionamiento aceptable.

En complemento a lo indicado, es importante mencionar que solo el 8 por ciento han hecho la apertura de nuevos puntos de venta, pero en los comentarios se referían a las razones y el temor por la apertura, esto demuestra que con financiamiento posiblemente tomarían riesgos para abrir un nuevo punto de venta.

La última interrogante viene a responder al objetivo de conocer las necesidades de financiamiento que tienen actualmente las pymes comerciales encuestadas, el 46 por ciento contestó que necesita el financiamiento para expandir el negocio, el 24 por ciento afirma que requiere financiamiento para comprar mercadería o inventarios, 16 por ciento para equipos de trabajo y 14 por ciento los utilizaría para vehículos de distribución.

En el corto plazo, los resultados describen las fuentes para el financiamiento de los recursos productivos que las pymes tienen a disposición y a partir de esta generar la creación de la estrategia, al preguntar sobre las fuentes financieras que disponen o utilizan estratégicamente las empresas, la mayoría contestó que cuentan con mercadería en consignación (70 por ciento), también en relación con los proveedores cuentan con periodos de pago de uno a tres meses (58 por ciento) y usan tarjetas de crédito (52 por ciento de los encuestados). Estos datos muestran la relevancia que tienen las relaciones con los proveedores por parte de las pymes comerciales para poder desarrollar su negocio.

Otro resultado de la encuesta es relacionado con el crecimiento a la fecha de estas pymes, la mayoría, es decir 56 por ciento de los consultados, afirmó que a través de las mismas ganancias que iba generando el negocio; también un 26 por ciento indicó que con el apoyo de los proveedores que entregan mercadería en consignación. Estos datos son indicadores importantes de la existencia de una adecuada administración financiera y control de los resultados, ratificando una estrategia de utilizar las ganancias para reinvertir en el propio negocio, y como afirmaron algunos encuestados, muchas veces las empresas no duran porque el dueño se gasta las ganancias en recursos personales 
y se olvida que el negocio debe mantenerse. Otros datos indican el 12 por ciento lo han hecho a través de ingresos de otro empleo y solo 6 por ciento a través de préstamos.

El tema de utilizar el financiamiento de las instituciones financieras, los resultados muestran que el 62 por ciento, sí estarían dispuestos al endeudamiento para hacer crecer su negocio, pero también cabe destacar que cuando se les pregunta sobre conocimientos en temas financieros muchos indican desconocimiento, el 52 por ciento de los encuestados indicaron estar en desacuerdo y totalmente en desacuerdo de conocer sobre temas financieros como leasing o renting y 44 por ciento afirmaron esta misma posición sobre el conocimiento de requerimientos para los créditos. Es decir, el desconocimiento origina las razones de temor por acceder al financiamiento que ofrece el mercado financiero a las pymes.

La consulta sobre los retos que enfrentan las pymes para lograr aplicar las estrategias de financiamiento, como se muestra en la figura 3 el resultado, es decir el 36 por ciento de los encuestados, indicaron estar ni en desacuerdo ni de acuerdo con que el mercado de Guanacaste es suficiente para que las pymes puedan desarrollarse, otros 46 por ciento encuestados sí se ubicaron entre de acuerdo y totalmente de acuerdo. Algunos encuestados comentaron que en Guanacaste el mercado es muy exigente y aunado a la situación económica actual del país no se visualiza un panorama de crecimiento económico para que los consumidores aporten tanto al desarrollo de las empresas comerciales.

Ante la consulta de la existencia de una capacitación suficiente y facilidades para obtener créditos, la mayoría indicó estar en desacuerdo con esa afirmación. Estos datos reafirman la información anterior, ya que los encuestados indicaron que tienen desconocimiento y también consideran que no hay capacitación y facilidades suficientes.

El mercado de Guanacaste es suficiente para que las pymes puedan desarrollarse.

Existe suficiente capacitación para las pymes en el tema de finanzas para el crecimiento de operaciones.

Considera que existen facilidades para obtener créditos en el sistema financiero nacional.

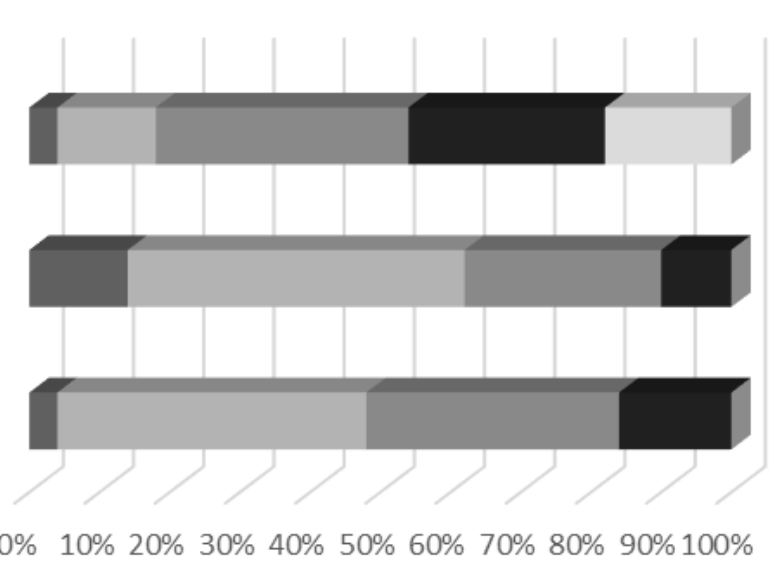

Totalmente en desacuerdo

De acuerdo

Figura 3. Percepción sobre los retos que enfrentan las pymes comerciales de Guanacaste para lograr aplicar las estrategias de financiamiento (Fuente: elaboración propia).

En la interrogante sobre cuál estrategia de negocio quisieran aplicar las pymes comerciales para el crecimiento de la empresa en el corto plazo, la mayoría, el 68 por ciento de los encuestados afirmó que quisieran abrir un nuevo punto de venta, el 54 por ciento afirmaron que quisieran 
aumentar sus inventarios. Lo que ratifica la información anterior relacionada con la apertura de negocios, porque son pocos los que han logrado abrir un nuevo punto de venta, y quizás la decisión de crecer por medio de esta estrategia se centraría en buscar los recursos necesarios por medio del financiamiento, y de este modo, desarrollar la empresa en nuevos puntos geográficos, a pesar de los comentarios que también aportaron sobre la situación coyuntural del mercado guanacasteco, aun así, los empresarios muestran deseos por crecer.

La encuesta termina con la pregunta sobre las estrategias de financiamiento que recomendarían para que las empresas de este sector usen en el corto plazo para el crecimiento de sus operaciones, las respuestas incluían que las empresas deben mejorar sus estrategias no solo financieras sino, también, en áreas como las ventas y mercadeo, capacitación en temas de aprender a buscar financiamiento, trabajar en encadenamientos para la reducción de costos y aumentar las posibilidades de ventas y desarrollo de mercado.

Adicionalmente, comentaron sobre fomentar asociaciones y cooperativas para mayor acceso a fondos cuando los emprendimientos se dedican a un mismo negocio, particularmente uno de los encuestados recomendó el uso de tarjetas de crédito indicando que si se paga en aproximadamente el mes de plazo no tiene cobro de intereses y aprovechar los descuentos y otros beneficios financieros.

\section{Conclusiones}

El análisis de las estrategias de financiamiento para la obtención de recursos productivos en las pequeñas y medianas empresas (pymes) comerciales de Guanacaste, muestra un panorama claro sobre las necesidades de financiamiento, fuentes de financiamiento y estrategias para el crecimiento y desarrollo de las pymes comerciales de Guanacaste.

Los recursos requeridos para la inversión inicial, la mayoría comenzaron sus negocios con ahorros personales o ingresos provenientes de otro empleo, el monto establecido fue menor a los cinco millones, posterior el crecimiento durante primer año se evidencio en la adquisición de más mercadería y contratación de personas.

Es recomendable que las pymes comerciales de Guanacaste realicen estudios detallados de su actividad económica, utilizando herramientas como planes de negocios, donde por ejemplo se analice el mercado para determinar la viabilidad financiera de la apertura de nuevos puntos de venta o la expansión de los negocios.

Las fuentes para el financiamiento de los recursos productivos que las pymes tienen a disposición para la creación de la estrategia, la investigación demuestra el eficiente uso de mercadería en consignación, se aprovechan los plazos de cancelación de tres meses otorgados por los proveedores, estos dos aspectos demuestran las buenas relaciones con los proveedores. A nivel mercado financiero se hace uso de las tarjetas de crédito y por último el uso de las ganancias de períodos anteriores, pero afirman tener desconocimiento en temas de financiamiento relacionado con deudas directas.

La recomendación para estas pymes comerciales de Guanacaste, es plantear capacitaciones sobre el uso de financiamiento ofrecido por el mercado financiero, mediante el apoyo de instituciones designadas por el gobierno para atender este sector económicas; Ministerio de Economía, Industria y Comercio, municipalidades locales, instituciones financieras con 
mecanismos de aprendizajes específicos en el tema, para lograr que estos empresarios sean capaces de generar escenarios comparativos con herramientas financieras de presupuestos y flujos de cajas que evalúan las opciones de un menor gastos de intereses, y así, mitigar el riesgo y temor de la decisión de expansión de los negocios.

Por último, estas empresas planean el crecimiento por medio de la expansión de abrir nuevos puntos de venta, aunque consideran un mercado Guanacasteco no tan seguro de aportar al crecimiento, pero algunos empresarios cuentan con ideas innovadoras para gestionar este crecimiento e incluyen las estrategias de financiamiento, ventas, encadenamientos productivos, aprendizaje continuo y la importancia de las capacitaciones en estos temas financieros.

Es recomendable que las empresas comprendan la importancia de la gestión empresarial en las diversas áreas de las pymes, abarcando las áreas de mercadeo, talento humano, operaciones, finanzas y otras particulares de cada empresa. La ejecución del crecimiento se debe sustentar sobre una estrategia integral de todas las áreas y acompañada de una educación financiera y de gestión empresarial sistemática, acciones que aumentan la posibilidad de crecimiento de las pymes y por ende el desarrollo económico y social de Guanacaste y el país.

Referencias bibliográficas

Carrasco, S. (2017). Servicios de Atención Comercial. Madrid: Paraninfo.

Chiavenato, I. (2017). Planeación estratégica (3a. Ed.). México: McGraw-Hill Interamericana.

Gutiérrez, T. (30 de enero, 2017). ¿Tiene una pyme? Esto es lo que ofrecen los bancos nacionales. La República. Recuperado de https://www.larepublica.net/noticia/-tiene-una-pyme-esto-eslo-que-ofrecen-los-bancos-nacionales

Harvard Business Review. (2017). Finanzas básicas. Guías HBR. Barcelona: Editorial Reverté.

Hernández, R.; Fernández, C. y Baptista, M. (2014). Metodología de la Investigación (Sexta Ed.). México: McGraw Hill.

Instituto Nacional de Estadísticas y Censos. (2019). Total, de empresas según provincia, cantón y distrito. DEE 2018. Recuperado de http://www.inec.go.cr/economia/directorio-de-empresasy-establecimientos

Ramírez, D. (2018). Contabilidad administrativa. (10a. Ed.). Ciudad de México: McGraw-Hill Interamericana.

Pymes Costa Rica. (2019). Conozca el tamaño de su empresa. ¿qué es una pyme? Ministerio de Economía, Industria y Comercio. Recuperado de http://pyme.go.cr/cuadro5.php?id=1

Pymes El Financiero. (13 de marzo, 2015). La 'muerte' de las pymes: ¿Cuánto tiempo duran los pequeños negocios y por qué? Estudios señalan que entre 70por ciento y 80por ciento de pequeños negocios duran menos de una década. El Financiero. Recuperado de https://www.elfinancierocr.com/pymes/la-muerte-de-las-pymes-cuanto-tiempo-duran-lospequenos-negocios-y-por-que/QMKIITYSUFENHADJA3UEFSQO5E/story/ 
InterSedes, $\mathbf{N}^{\circ}$ 42. Vol XX (2019). ISSN 2215-2458

Ross, S. A., Westerfield, R. W., Jaffe, J. y Jordan, B. D. (2018). Finanzas corporativas. (11a. Ed.). Ciudad de México: McGraw-Hill Interamericana.

Salas, T. (2016). Análisis y diagnóstico financiero. (5ta Ed.). San José: Ediciones Guayacán.

Thompson, A. A., Strickland III, A. J., Janes, A., Sutton, C., Peteraf, M. A. y Gamble, J. E. (2018). Administración estratégica (2a. ed.). Ciudad de México: McGraw-Hill Interamericana. 